\title{
The effect of HIV infection on overdose mortality
}

\author{
Cunlin Wanga , David Vlahov ${ }^{a, b}$, Noya Galai ${ }^{a}$, Stephen R. Cole ${ }^{a}$, \\ Joseph Bareta ${ }^{a}$, Robin Pollini ${ }^{a}$, Shruti H. Mehta ${ }^{a}$, Kenrad E. Nelson ${ }^{a}$ \\ and Sandro Galea ${ }^{b}$
}

\begin{abstract}
Objectives: To quantify the association of HIV infection with overdose mortality and explore the potential mechanisms.

Design: A prospective cohort study.

Methods: A total of 1927 actively injecting drug users who were HIV seronegative at baseline, of whom 308 later HIV seroconverted, were followed semi-annually for death from 1988 to 2001. Survival analyses using marginal structural and standard Cox models were used to evaluate the effect of HIV infection on the risk of overdose mortality.

Results: Overdose death rates were higher in HIV-seropositive than HIV-seronegative drug users: 13.9 and 5.6 per 1000 person-years, respectively $(P<0.01)$. The hazard ratio $(\mathrm{HR})$ was 2.54 [95\% confidence interval $(\mathrm{Cl}) 1.47,4.38]$ for the marginal structural model and $2.06(95 \% \mathrm{Cl} 1.25,3.38)$ for the standard Cox model, both adjusted for demographics, drug injection characteristics, alcohol abuse, substance abuse treatment, and sexual orientation. Adjusting for possible time-varying mediators (i.e. drug use, medical conditions and healthcare access) in extended marginal structural models reduced the effect of HIV on overdose mortality by $30 \%(\mathrm{HR} 1.82,95 \% \mathrm{CI} 1.01,3.30)$. Abnormal liver function was associated with a higher risk of overdose mortality (HR 2.00, 95\% $\mathrm{Cl} 1.05,3.84)$; adjustment for this further reduced the effect of HIV on overdose mortality.

Conclusion: HIV infection was associated with a higher risk of overdose mortality. Drug use behavior, systematic disease and liver damage associated with HIV infection appeared to account for a substantial portion of this association. The data suggest a group to target with interventions to reduce overdose mortality rates.
\end{abstract}

(c) 2005 Lippincott Williams \& Wilkins

AIDS 2005, 19:935-942

Keywords: HIV, injection drug user, mortality, overdose

\section{Introduction}

Among injection drug users (IDU), two commonly reported causes of death include drug overdose, and in HIV epidemic areas, AIDS. Whereas the HIV epidemic among this group has attracted substantial attention over the past two decades, in the United States, Europe and Australia, drug overdose and related deaths have also been consistently increasing in recent years [1-4]. Meanwhile, data from most western European countries and north America have shown that the number of individuals using opiates and cocaine has either been stable or declined in the 1990s [5]. Moreover, this trend of increase in overdose deaths was also observed within drug user cohorts [6-9].

Interestingly, cohort studies have also shown that the increase in overdose deaths paralleled the increase in AIDS mortality [6,8-10]. So far, only a few studies have

From the ${ }^{\mathrm{a}}$ Department of Epidemiology, Johns Hopkins Bloomberg School of Public Health, Baltimore, MD, USA, and the ${ }^{\mathrm{b}}$ Center for Urban Epidemiologic Studies, New York Academy of Medicine, New York, NY, USA.

Correspondence to Dr David Vlahov, New York Academy of Medicine, 1216 Fifth Avenue, New York, NY 10029, USA.

E-mail: dvlahov@nyam.org

Received: 2 July 2004; revised: 27 January 2005; accepted: 28 January 2005. 
addressed this association of HIV and overdose mortality [6,7,9,11-14]. These studies have been limited by: (i) including mostly HIV-prevalent drug users at baseline whose date of HIV seroconversion was unknown; (ii) failing to adjust for important confounders that can be associated with both HIV infection and drug overdose; or (iii) having either a small sample or a short follow-up period, therefore reporting only a few events.

Meanwhile, the mechanism of fatal overdose remains unclear [2,15-17]. Progress from recent research has provided more explanations on the possible mechanism of drug overdose, which included loss of tolerance, metabolic variation, interaction of poly-drug use, and systematic disease $[2,4,18]$. These data have revealed characteristics that can serve as the basis for hypothesizing pathways to explore the association of HIV infection and overdose death.

The first hypothesis for an HIV and overdose association is a reduction in drug tolerance as a result of the effects of HIV infection. Overdose deaths occur at greater frequency when drug users are discharged from drug treatment or released from prison, presumably because of relapse using similar doses but with less tolerance. For $\mathrm{HIV}$, as infection progresses, symptoms can interfere with drug use patterns, resulting in reduced, or intermittent use, during which tolerance subsides. A resumption of use, especially with doses used when more tolerant, could be a possible consideration in overdose mortality. Second, for opiate users, drug-induced respiratory depression is probably a primary reason for overdose death. In combination with current or previous pulmonary disease, which is more common in HIV-seropositive drug users than seronegative drug users, overdose may reflect an underlying susceptibility to respiratory depression among HIV-infected drug users, especially in those with advanced immunosuppression [19]. Third, hepatic diseases may also be associated with a higher risk of overdose mortality by affecting opiate metabolism. The prevalence of hepatitis $\mathrm{C}$ virus (HCV) infection among IDU is high, varying from 60 to $90 \%$ [20-22]. Studies have shown that the progression of $\mathrm{HCV}$ can be accelerated in those with HIV infection [23-25], which may, in turn, impair the liver's capability to metabolize morphine. Fourth, damage of the central nervous system, which can be caused by drugs, HIV infection and head trauma associated with violence among drug users [26,27], may increase the vulnerability of the inhibitory effects of opiates on the respiratory system [6], or impair their cognitive function, which can affect the ability to judge risky drug use behavior. Fifth, damage of immune system can undermine the immune response of chronic drug users to produce moderate affinity antibodies and reduce the circulating free fraction of active heroin metabolites [28].

In this study we explored the effect of HIV infection on the risk of overdose mortality and the potential mechanisms for this association among 1927 active IDU, and compared HIV seroconverters with HIVnegative drug users on overdose mortality over a period of 13 years.

\section{Materials and methods}

\section{Study population}

Participants were enrolled in the AIDS Linked to the Intravenous Experience (ALIVE) study in Baltimore, MD, USA [29]. IDU were recruited through community outreach from 1988 to 1989, with additional recruitment in 1994 and 1998. All participants were followed with semi-annual visits that included a questionnaire interview that obtained information for the preceding 6 months on sexual behavior, drug use, medical history, and antiretroviral treatment if HIV seropositive, a clinical examination, and venipuncture for laboratory tests including CD4 cell count and liver function.

\section{Ascertainment of overdose death}

Death was ascertained through the National Death Index (NDI), with confirmation through death certificates and chief medical examiner reports. This study was truncated at December 2001 because of the approximate 2 year lag period from the NDI. Overdose mortality was determined in two ways. Approximately $60 \%$ of overdose deaths in the ALIVE study were confirmed by autopsy reports. For deaths for which autopsy reports were unavailable, overdose death was determined when it was the only cause of death from NDI-Plus, and the death certificate as well as supplemental medical records were reviewed by a clinical endpoints committee. In the study, after excluding overdose deaths that occurred 2 years after the last follow-up visit, there was a total of 92 overdose deaths, of whom 67 were HIV seropositive.

\section{Laboratory assays}

HIV antibodies were assayed using commercial tests and were interpreted using standard criteria. T-cell subsets were determined by flow cytometry. Abnormal liver function was determined by commercial assay, and defined as being out of the normal range for both total serum bilirubin $(0.1-1.0 \mathrm{mg} / \mathrm{dl})$ and serum albumin $(3.5-5.0 \mathrm{~g} / \mathrm{dl})[30,31]$.

\section{Statistical analysis}

Mortality rates were calculated as the number of deaths divided by the number of person-years. Person-years was determined from the date of staggered entry [32] to the date of death, dropout, or the date of administrative censoring. The censoring can be the end of the study, 31 December 2001, the date of the initiation of highly active antiretroviral therapy defined by the International AIDS Society guidelines [33], the date of the last follow-up visit if the visit was more than one year before 31 December 
2001 , or if the date of death was more than 2 years after the last follow-up. The complement of the extended Kaplan-Meier and log-rank test were used to compare the time to overdose death between HIV seroconverted and seronegative groups. The extended Kaplan-Meier allows HIV seroconverters to contribute person-time to the HIV-1-seronegative group before seroconversion via late entries into the HIV seroconverters group [32,34].

The marginal structural Cox regression models [35,36] were used to estimate the total effect of HIV infection on overdose mortality, accounting for factors potentially associated with both HIV seroconversion and overdose mortality, but could also be affected by HIV infection. In our analysis, at the univariate model, we considered all collected variables including demographics, social economics status, drug abuse behavior (including both inhaled or ingested use: crack, heroin, cocaine, marijuana, street methadone; injection drug use: type of drug injected, injection frequency, needle sharing, use of shooting gallery; and drug abuse treatment: detoxification or methadone programme enrollment), alcohol abuse and sexual behavior. Factors identified as potentially significant $(P<0.1)$ were included into the multivariate model. As described elsewhere [35-37], marginal structural models weight person-time proportionally to the conditional probability of exposure given timedependent confounders. The weights are a generalization of Horwitz-Thompson weights, and are generated using the same method used to create the propensity score. The weighted data reflect a pseudo-population in which the time-varying covariates do not predict HIV seroconversion, but the impact of HIV infection on the hazard of overdose death is still the same as in the original population. Therefore, the weighted data remove the association of confounders with HIV seroconversion, but preserve the association of HIV and overdose deaths. We also stabilized the weights by baseline characteristics, and subsequently included these baseline characteristics in the marginal structural model. In addition, we also fit standard time-dependent Cox regression models for comparisons with marginal structural models. Robust variances were estimated to account for the correlation between observations from the same participants.

Although not a perfect method [38], previous studies have suggested that to explore the mechanism of exposure effect on outcome, time-dependent covariates that may serve as 'intermediates' can be added into the model to see the corresponding change on the association between exposure and outcome [39]. This type of analysis has been applied in studies to identify mediators [40] and surrogate endpoints [41]. In the present analysis, to explore potential time-varying mediators that lie on a pathway between HIV infection and overdose mortality, we compared the total effect of HIV on overdose mortality from the standard marginal structural model to an approximated direct effect of HIV on overdose mortality that was not through the time-varying mediator, and was estimated from a marginal structural model with that time-varying mediator included as a covariate. The direct effect was approximated by assuming that there were no confounders of the mediators and overdose mortality. The mediators we explored included drug and alcohol abuse characteristics, substance abuse treatment, medical conditions, healthcare access and abnormal liver function.

\section{Results}

\section{Baseline characteristics}

The study consisted of 1927 participants who were actively using drugs during the 6 months before the baseline. Among them, 1619 were persistently HIV seronegative and $308 \mathrm{HIV}$ seroconverted during the follow-up. At baseline, participants who later HIV seroconverted were more likely than persistently seronegative drug users to be younger (median age 31.0 versus 35.1 years, $P<0.01$ ), to be black; and were less likely to be educated, or to be ever married (Table 1). In addition, HIV seroconverters were more likely than uninfected drug users to have initiated drug use at an earlier age, to have used heroin, and to have a homosexual orientation, but were less likely to report previous methadone treatment. There were no significant differences between the two groups with respect to the other factors.

\section{Predictors of HIV seroconversion}

As shown in Table 2, HIV seroconversion was positively associated $(P<0.05)$ with younger age at baseline, younger age of drug initiation, injecting drugs, and male homosexuality, and to a lesser degree with shooting gallery use $(P<0.07)$; but was inversely associated $(P<0.05)$ with marijuana use, methadone treatment, and detoxification; and marginally associated with employment $(P=0.06)$. Gender, race, education, marital status, or needle sharing did not discriminate HIV seroconversion.

\section{Overall effect of HIV infection on overdose mortality}

As shown in Table 3, there was a total of 13871 personyears (PY), with a median follow-up of 6.6 years for HIV-seronegative and 5.9 years for HIV-seropositive participants. With a total of 92 validated overdose deaths, of which 69 were HIV seropositive (Table 3), the overall overdose mortality rate was 6.6/1000 PY [95\% confidence interval (CI) 5.4, 8.1/1000 PY], with 5.6/1000 PY (95\% CI 4.4, 7.1/1000 PY) for HIV-seronegative drug users and 13.9/1000 PY (95\% CI 9.4, 20.5/1000 $\mathrm{PY})$ for HIV-seropositive drug users $(P<0.01)$. Figure 1 shows the complement of extended Kaplan-Meier on the probability of overdose mortality by HIV infection status through the follow-up, which was higher for 
Table 1. Baseline information of injection drug users by HIV seroconversion status in Baltimore, Maryland $1988-2001$.

\begin{tabular}{|c|c|c|c|c|c|}
\hline \multirow[b]{2}{*}{ Baseline characteristic } & \multicolumn{2}{|c|}{ HIV seronegative $(n=1619)$} & \multicolumn{2}{|c|}{ HIV seropositive $(n=308)$} & \multirow[b]{2}{*}{$P$ value } \\
\hline & $N$ & Proportion (\%) & $N$ & Proportion (\%) & \\
\hline \multicolumn{6}{|l|}{ Demographic } \\
\hline \multicolumn{6}{|l|}{ Age (years) } \\
\hline$<30$ & 347 & 21.4 & 117 & 38.0 & \\
\hline $30-40$ & 824 & 50.9 & 152 & 49.4 & \\
\hline $40-50$ & 389 & 24.0 & 34 & 11.0 & \\
\hline$>50$ & 59 & 3.6 & 5 & 1.6 & $<0.01$ \\
\hline Male education & 1202 & 75.1 & 228 & 74.0 & 0.66 \\
\hline Less than high school & 859 & 53.7 & 193 & 62.7 & \\
\hline High school & 342 & 21.4 & 55 & 17.9 & \\
\hline Some college or more & 398 & 24.9 & 60 & 19.5 & 0.04 \\
\hline Employed & 295 & 18.5 & 64 & 20.8 & 0.35 \\
\hline Married or ever & 613 & 38.4 & 95 & 30.8 & 0.01 \\
\hline Black race & 1471 & 91.4 & 290 & 94.5 & 0.07 \\
\hline \multicolumn{6}{|l|}{ Drug use/treatment characteristic } \\
\hline \multicolumn{6}{|l|}{ Age of first drug use (years) } \\
\hline$<18$ & 520 & 32.8 & 123 & 40.2 & \\
\hline $18-25$ & 677 & 42.6 & 116 & 37.9 & \\
\hline$>25$ & 391 & 24.6 & 67 & 21.9 & 0.04 \\
\hline \multicolumn{6}{|l|}{ Injection pattern } \\
\hline No injection & 253 & 16.4 & 48 & 15.9 & \\
\hline$\leq 1 /$ day & 521 & 33.8 & 106 & 35.1 & \\
\hline$>1 /$ day & 768 & 49.8 & 148 & 49.0 & 0.9 \\
\hline \multicolumn{6}{|l|}{ Type of drug use } \\
\hline Not injection & 253 & 16.5 & 48 & 16.0 & \\
\hline Cocaine & 152 & 9.9 & 8 & 2.7 & \\
\hline Heroin & 141 & 9.2 & 41 & 13.6 & \\
\hline Cocaine and heroin & 989 & 64.4 & 204 & 67.8 & $<0.01$ \\
\hline Shared needles (yes) & 845 & 53.3 & 148 & 48.5 & 0.12 \\
\hline Shooting gallery use (yes) & 629 & 39.7 & 108 & 35.4 & 0.16 \\
\hline Methadone treatment (yes) & 154 & 10.3 & 16 & 5.4 & 0.01 \\
\hline Detoxification programme (yes) & 206 & 13.7 & 36 & 12.2 & 0.50 \\
\hline \multicolumn{6}{|l|}{ Other } \\
\hline \multicolumn{6}{|l|}{ Health insurance } \\
\hline No & 575 & 37.4 & 105 & 35.1 & \\
\hline Public insurance & 787 & 51.2 & 170 & 56.9 & \\
\hline Private insurance & 174 & 11.3 & 24 & 8.0 & 0.11 \\
\hline Ever in jail (yes) & 223 & 21.9 & 63 & 23.3 & 0.63 \\
\hline \multicolumn{6}{|l|}{ Sexual orientation } \\
\hline Heterosexual & 1418 & 92.1 & 262 & 86.8 & \\
\hline Male homosexual & 80 & 5.2 & 33 & 10.9 & \\
\hline Female homosexual & 41 & 2.7 & 7 & 2.3 & $<0.01$ \\
\hline
\end{tabular}

HIV-seropositive than for uninfected participants $(\log$ rank test $P=0.03$ ).

Table 3 also shows the hazard ratio (HR) of overdose mortality by HIV infection serostatus from crude, adjusted and weighted models, respectively. The unadjusted HR of overdose death for HIV-seropositive versus uninfected participants was 2.04. With the marginal structural model adjusting for confounders associated with both HIV seroconversion and overdose mortality, the HR of overdose death by HIV status was 2.54. Using the standard time-dependent Cox model, the HR was 2.06 , representing approximately a $20 \%$ reduction between weighted and adjusted models on the risk of overdose mortality by HIV status.

\section{Mechanism of the effect of HIV infection on overdose mortality}

Table 4 shows a comparison of the results from the standard marginal structural model with the results of extended marginal structural models that include potential mediators as time-varying covariates. Each of the following: injection frequency (12\%), use of shooting gallery $(13 \%)$, needle sharing (13\%), type of drugs injected $(12 \%)$, employment status $(12 \%)$ was associated with more than a $10 \%$ reduction on the association of HIV infection and overdose death. Daily alcohol use was associated with only a $5 \%$ reduction in the association of HIV and overdose death. In model 1 , adjusting for drug use, daily alcohol use and employment reduced the association of HIV infection and overdose death by approximately 15\%: the HR decreased from 2.54 in the standard marginal structural model to 2.18 in the extended marginal structural model 1. Among medical conditions and healthcare factors, shortness of breath, diarrhea, and a recent inpatient visit were each associated with a $10 \%$ reduction on the association of HIV infection and overdose death, whereas health insurance was associated with a 5\% reduction on the association of HIV infection and overdose death. Adjusting for 
Table 2. Characteristics associated with HIV seroconversion for 1927 active drug users in Baltimore, Maryland 1988-2001.

\begin{tabular}{|c|c|c|}
\hline Characteristic & Adjusted RR & $95 \% \mathrm{Cl}$ \\
\hline \multicolumn{3}{|l|}{ Age (years) } \\
\hline $30-40$ versus $<30$ & 0.61 & $0.44,0.84$ \\
\hline $40-50$ versus $<30$ & 0.38 & $0.23,0.63$ \\
\hline \multirow[t]{2}{*}{$>50$ versus $<30$} & 0.45 & $0.16,1.32$ \\
\hline & 0.96 & $0.68,1.35$ \\
\hline \multicolumn{3}{|l|}{ Female education } \\
\hline $\begin{array}{l}\text { High school versus } \\
\text { less than high school }\end{array}$ & 0.81 & $0.57,1.17$ \\
\hline $\begin{array}{l}\text { College versus } \\
\text { less than high school }\end{array}$ & 0.85 & $0.59,1.21$ \\
\hline Marriage & 1.05 & $0.76,1.45$ \\
\hline Black & 0.88 & $0.49,1.59$ \\
\hline \multicolumn{3}{|l|}{ Age of first drug use (years) } \\
\hline $18-25$ versus $<18$ & 0.69 & $0.50,0.94$ \\
\hline$>25$ versus $<18$ & 0.83 & $0.57,1.20$ \\
\hline \multicolumn{3}{|l|}{ Time-varying covariates } \\
\hline Employment & 0.72 & $0.51,1.02$ \\
\hline Marijuana use & 0.71 & $0.52,0.97$ \\
\hline $\begin{array}{l}\text { Drug injection less than } \\
\text { daily versus no }\end{array}$ & 1.49 & $0.98,2.25$ \\
\hline Daily injection versus no & 1.76 & $1.16,2.66$ \\
\hline Needle sharing & 1.04 & $0.75,1.45$ \\
\hline Shooting gallery use & 1.45 & $0.94,2.25$ \\
\hline Methadone treatment & 0.38 & $0.18,0.81$ \\
\hline Detoxification programme & 0.55 & $0.32,0.94$ \\
\hline Daily alcohol use & 1.15 & $0.82,1.62$ \\
\hline Male homosexuality & 2.14 & $1.34,3.40$ \\
\hline
\end{tabular}

$\mathrm{Cl}$, Confidence interval; RR, risk ratio.

shortness of breath, diarrhea, a recent inpatient visit, and health insurance reduced the association of HIV infection and overdose by approximately $20 \%$ : from a HR of 2.54 in the standard marginal structural model to 2.06 (95\% CI $1.17,3.61)$. As shown in model 2 , adjusting for both drug use characteristics and medical conditions reduced the association of HIV infection and overdose by almost 30\%, from an $\mathrm{HR}$ of 2.54 in the standard marginal structural model to $1.82(95 \%$ CI $1.01,3.30, P=0.05)$ in the extended marginal structural model 2 .

\section{The association of abnormal liver function with overdose mortality}

Overall, 1326 participants (69\%) had liver function tests. Adjusting for abnormal liver function in the marginal structural model reduced the association of HIV and overdose death by $12 \%$. Adjusting for drug use characteristics, medical conditions, healthcare, and abnormal liver function together reduced the association between HIV and overdose death by $35 \%$, with a decrease in the HR from 3.07 (95\% CI 1.52, 6.18, $P=0.002)$ in the standard marginal structural model to 2.01 (95\% CI, $0.96,4.20, P=0.06)$ in the extended marginal structural model. Also, abnormal liver function itself was associated with a higher risk of overdose mortality (HR 2.00, 95\% CI $1.05,3.84, P=0.04)$, by adjusting for drug and alcohol use, drug abuse treatment, employment, medical condition, and health insurance.

In addition, among participants who died from overdoses and had autopsy reports $(n=51)$, at the body examination, the median level of blood morphine, the proportion with detected alcohol use, and the proportion of detected multidrug use for HIV-seropositive and HIV-seronegative drug users was 533 and $699 \mu \mathrm{g} / 1(P=0.62) ; 54.6$ and $48.5 \%(P=0.73)$, and 81.8 and $96.8 \%(P=0.1)$, respectively.

\section{Discussion}

The major finding of this study was the observation of a higher rate of overdose mortality among those with HIV infection. Factors accounting for approximately $35 \%$ of the association between HIV infection and overdose death included a combination of drug use behavior, medical conditions, healthcare utilization and abnormal liver function.

Our data are in contrast to a few previous studies that have failed to identify a significant association of HIV infection and overdose mortality. The COMCAT study from Italy compared 474 HIV-prevalent IDU with 1958 IDU with unknown HIV serostatus, followed from 1980 to 1991, on the cause of mortality, and found no effect of HIV infection on overdose death [6]. However, the generalizability of the study was limited because: (i) hospitaltraced prevalent rather than incident cases were used; (ii) the control group did not distinguish serostatus and probably included HIV-seropositive IDU; and (iii) the comparison was based on an unadjusted mortality rate. Another study from New York City consisted of 318

Table 3. Association of HIV on overdose mortality determined by marginal structural model and standard Cox model among 1927 active drug users in Baltimore, Maryland 1988-2001.

\begin{tabular}{lcccccc}
\hline Exposure & No. of OD & Person-years & Mortality rate $(/ 1000 \mathrm{PY})$ & Crude HR $(95 \% \mathrm{Cl})^{\text {Adjusted }^{\mathrm{a}}(95 \% \mathrm{Cl})}$ & Weighted $^{\mathrm{a}}(95 \% \mathrm{Cl})$ \\
\hline HIV seronegative & 67 & 12067 & $5.6(4.4,7.1)$ & 1 & 1 \\
HIV seropositive & 25 & 1804 & $13.9(9.4,20.5)$ & $2.04(1.28,3.24)$ & $2.06(1.25,3.38)$ & $2.54(1.47,4.38)$ \\
Total & 92 & 13871 & $6.6(5.4,8.1)$ & &
\end{tabular}

$\mathrm{Cl}$, Confidence interval; $\mathrm{HR}$, hazard ratio; $\mathrm{OD}$, overdose death; PY, person-years.

a Both the adjusted and the weighted models accounted for the same set of covariates: age, sex, race, marital status, education level, employment, and age of drug initiation, injection frequency, needle sharing, and use of shooting gallery at baseline; as well as time-varying employment, drug injection and frequency, detoxification programme, methadone treatment, needle sharing, shooting gallery, alcohol use and homosexual orientation. 


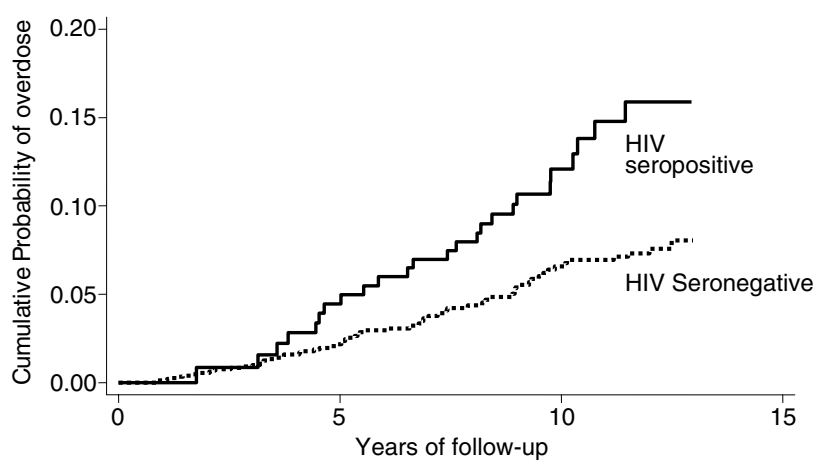

Fig. 1. Complement of Kaplan-Meier survival estimates by HIV status.

HIV-seropositive (14 were HIV seroconverters) and 411 HIV-seronegative IDU followed for a median of 3 years [12]. Comparisons of crude overdose mortality rates did not differ by HIV serostatus, but only three overdose deaths were identified in the HIV-seropositive group. Among studies that have identified a positive association between HIV infection and overdose death, one study had a similar result to ours [13], another observed a crude relative risk of 3.6 [11], and three additional studies $[7,9,14]$ suggested a positive but lower magnitude of effect of HIV infection on overdose mortality than that reported here.

The mechanism of the impact of HIV infection on drug overdose was explored through three hypotheses in this study. First, drug use behavior may affect both HIV risk and overdose, thus working as a confounder, but alternatively HIV infection could affect drug use (i.e. reduced use with HIV symptoms affecting tolerance to drugs or cognitive function), which in turn could result in a higher risk of overdose death. Studies have shown that after HIV infection, drug users tend to reduce their drug use [43] and risky sexual behavior [44], are less likely to go to a shooting gallery [45] and share needles [43], but are also more likely to seek medical care [46] and enroll into drug treatment [47]. Studies have also shown that drug users who died from (or experience non-fatal) overdoses were individuals who had relapsed after ceased or reduced drug use before the event $[16,48]$. Furthermore, drug users who inject drugs in an environment in which they have not used drugs before have decreased tolerance and a higher risk of overdose mortality [18]. Drug users who are enrolled in a drug treatment programme may also have a higher risk of overdose as a result of reduced tolerance that is associated with abstinence or infrequent drug use during treatment $[18,49]$. In our study, adjusting for drug injection frequency, the type of drug injected, the use of a shooting gallery, and needle sharing reduced the association of HIV and overdose death by approximately $15 \%$ in the extended marginal structural model. Also, the results from autopsy reports showed lower morphine levels and less multidrug use among HIV-seropositive than seronegative drug users, suggesting that it is not the dose that defines 'overdose', but rather these other factors.

Second, HIV infection can undermine the immune system and cause multisystem disorders. Certain systematic diseases can be associated with a higher risk of overdose mortality. Among them, pulmonary disease and respiratory infection are two of the most plausible diseases, because drug users with decreased pulmonary function may be at increased vulnerability to fatal respiratory depression, and therefore at a greater risk of overdose death [2]. In our study, adjusting for shortness of breath, diarrhea, a recent inpatient visit, and health insurance status reduced the association of HIV and overdose mortality by $20 \%$. Both shortness of breath and

Table 4. Association of HIV on overdose mortality adjusting for possible mediators in extended marginal structural models among 1927 injection drug users in Baltimore, Maryland 1988-2001.

\begin{tabular}{|c|c|c|c|c|}
\hline \multirow[b]{2}{*}{ Characteristics } & \multicolumn{2}{|c|}{ Model 1} & \multicolumn{2}{|c|}{ Model 2} \\
\hline & $\mathrm{HR}$ & $95 \% \mathrm{Cl}$ & HR & $95 \% \mathrm{Cl}$ \\
\hline HIV & 2.18 & $1.22,3.89$ & 1.82 & $1.01,3.30$ \\
\hline Injection heroin versus no injection & 5.50 & $2.85,10.61$ & 5.11 & $2.48,10.52$ \\
\hline Injection cocaine versus no injection & 2.03 & $0.58,7.08$ & 1.67 & $0.46,6.13$ \\
\hline Both heroin and cocaine versus no injection & 4.77 & $2.50,9.08$ & 4.28 & $2.09,8.75$ \\
\hline Daily injection & 0.69 & $0.43,1.11$ & 0.68 & $0.41,1.13$ \\
\hline Needle sharing & 1.04 & $0.64,1.69$ & 0.96 & $0.59,1.54$ \\
\hline Shooting gallery & 0.43 & $0.13,1.41$ & 0.42 & $0.13,1.36$ \\
\hline Employment & 0.57 & $0.30,1.09$ & 0.60 & $0.30,1.20$ \\
\hline Diarrhea & - & - & 2.03 & $0.82,5.00$ \\
\hline Shortness of breath & - & - & 1.61 & $0.75,3.42$ \\
\hline Inpatient visit & - & - & 2.09 & $1.24,3.53$ \\
\hline
\end{tabular}

$\mathrm{Cl}$, Confidence interval; $\mathrm{HR}$, hazard ratio.

${ }^{a}$ Both models were extended marginal structural models with the 'mediators' added as time-varying covariates. Daily alcohol, methadone treatment and detoxification were included in both models, and health insurance was included in model 2 to control for confounding between mediators and overdose deaths [42]. 
diarrhea tended to be associated with a higher risk of overdose mortality. The association between diarrhea and overdose death may be associated with the overall physical health condition, or be an indicator of drug use withdrawal symptoms.

Third, hepatic disease can impact the overdose mortality through prolonging the metabolism of opiates. HIV infection that is associated with the acceleration of HCV progression may affect overdose mortality through accelerated liver damage, therefore increasing vulnerability to fatal respiratory depression. Studies have shown that co-infection of HIV among HCV patients has resulted in a higher level of $\mathrm{HCV}$ viral load as a result of damaged cellular immunity [50,51], resulting in significantly accelerated liver fibrosis and cirrhosis, a poorer response to interferon therapy and increased liver necroinflammatory lesions, as well as resulting in an increased risk of liver-related mortality [23-25,51]. This effect has been shown to be more apparent among HIVinfected individuals who have lower CD4 cell levels $[23,24]$, which is different from our sample that consisted of HIV seroconverters. Given the median 8-10 years latent period of HIV infection and the median 5.9 years of follow-up for HIV seroconverters in our study, the impact of HIV infection on overdose mortality through accelerating liver function damage may be moderate.

Several study limitations should be acknowledged. The small number of overdose deaths identified among the HIV seroconverters identified during follow-up has limited our ability to explore another hypothesis: the higher risk of overdose death among HIV-seropositive drug users may be associated with progressed immunodeficiency. A separate analysis that includes both HIVprevalent drug users and HIV-incident drug users may be able to address this hypothesis. This can be further considered by investigating whether highly active antiretroviral therapy is associated with a lower risk of overdose mortality by adjusting for treatment access factors. Also, we did not have mental health information about the participants during the follow-up; therefore, we were unable to explore the hypothesis that HIV infection may affect the mental health condition and therefore increase the risk of overdose mortality, which has been shown in recent studies [52,53]. In addition, our population was limited to IDU; the association of HIV and overdose deaths specifically in a population of noninjectors merits further study.

In summary, our study demonstrates that HIV infection and abnormal liver function is associated with a higher risk of overdose mortality among active drug users. Drug use behavior, systematic disease and liver function damage associated with HIV infection accounted for approximately $35 \%$ of the effect of HIV on overdose deaths. These findings suggest that special attention about overdose prevention should be directed to individuals with HIV infection who use illicit drugs. Potential interventions include counseling at post-HIV testing counseling, closer monitoring and easier access of drug abuse treatment to drug users with HIV infection, and in cities where naloxone is available, training those with authority to prescribe or administer naloxone to drug users, which has been shown substantially to reduce overdose morbidity and mortality [54].

\section{Acknowledgements}

The authors would like to thank David Thomas, MD, for liver function tests, Joseph B. Margolick, MD, $\mathrm{PhD}$, for T-cell subset studies, and Lisette Johnson and Lisa Purvis for project management.

Sponsorship: This study is supported by the National Institute on Drug Abuse grants DA 04334 and DA12568. Dr Wang is also partly supported by Fogarty International Center/USNIH (2 D 43TW000010AITRP, Johns Hopkins University).

The authors have no potential conflicts, financial or otherwise.

\section{References}

1. Hickman M, Madden P, Henry I, Baker A, Wallace C, Wakefield $\mathrm{J}$, et al. Trends in drug overdose deaths in England and Wales 1993-98: methadone does not kill more people than heroin. Addiction 2003; 98:419-425.

2. Warner-Smith $M$, Darke S, Lynskey M, Hall W. Heroin overdose: causes and consequences. Addiction 2001; 96:11131125.

3. Preti $A$, Miotto $P$, De Coppi $M$. Deaths by unintentional illicit drug overdose in Italy, 1984-2000. Drug A/cohol Depend 2002; 66:275-282.

4. Darke S, Hall W. Heroin overdose: research and evidencebased intervention. J Urban Health 2003; 80:189-200.

5. United Nations. Global illicit drug trends, 2003. Vienna, Austria: United Nations Office of Drugs and Crime; 3003. pp. $101-135$

6. Galli M, Musicco M. Mortality of intravenous drug users living in Milan, Italy: role of HIV-1 infection. COMCAT Study Group. AIDS 1994; 8:1457-1463.

7. Vlahov D, Tang A, Lyles C, Rezza G, Thomas D, Cohn S. Increased frequency of overdose deaths among HIVinfected injection drug users. Addiction Res 2000; 8:311326.

8. Borrell C, Pasarin MI, Cirera E, Klutke P, Pipitone E, Plasencia $A$. Trends in young adult mortality in three European cities: Barcelona, Bologna and Munich, 1986-1995. J Epidemiol Commun Health 2001; 55:577-582.

9. Zaccarelli $M$, Gattari $P$, Rezza G Conti S, Spizzichino L Vlahov $D$, et al. Impact of HIV infection on non-AIDS mortality among Italian injecting drug users. AIDS 1994; 8:345350 .

10. de la Fuente L, Barrio G, Vicente J, Bravo MI, Santacreu J. The impact of drug-related deaths on mortality among young adults in Madrid. Am J Public Health 1995; 85:102-105.

11. van Haastrecht $H$ J, van Ameijden EJ, van den Hoek JA, Mientjes GH, Bax JS, Coutinho RA. Predictors of mortality in the Amsterdam cohort of human immunodeficiency virus (HIV)-positive and HIV-negative drug users. Am J Epidemiol 1996; 143:380-391. 
12. Selwyn PA, Alcabes P, Hartel D, Buon D, Schoenbaum EE, Klein RS, et al. Clinical manifestations and predictors of disease progression in drug users with human immunodeficiency virus infection. $N$ Engl / Med 1992; 327:16971703.

13. van Ameijden EJ, Langendam MW, Coutinho RA. Dose-effect relationship between overdose mortality and prescribed methadone dosage in low-threshold maintenance programs. Addict Behav 1999; 24:559-563.

14. Eskild A, Magnus P, Samuelsen SO, Sohlberg C, Kittelsen P. Differences in mortality rates and causes of death between HIV positive and HIV negative intravenous drug users. Int J Epidemiol 1993; 22:315-320.

15. Darke S, Zador D. Fatal heroin 'overdose': a review. Addiction 1996; 91:1765-1772.

16. Tagliaro F, De Battisti Z, Smith FP, Marigo M. Death from heroin overdose: findings from hair analysis. Lancet 1998; 351:1923-1925.

17. Darke S, Hall W, Kaye S, Ross J, Duflou J. Hair morphine concentrations of fatal heroin overdose cases and living heroin users. Addiction 2002; 97:977-984.

18. White JM, Irvine RJ. Mechanisms of fatal opioid overdose. Addiction 1999; 94:961-972.

19. van Ameijden EJ, Krol A, Vlahov D, Flynn C, van Haastrecht HJ, Coutinho RA. Pre-AIDS mortality and morbidity among injection drug users in Amsterdam and Baltimore: an ecological comparison. Substance Use Misuse 1999; 34:845865 .

20. Diamantis I, Bassetti S, Erb P, Ladewig D, Gyr K, Battegay M. High prevalence and coinfection rate of hepatitis $G$ and $C$ infections in intravenous drug addicts. J Hepatol 1997; 26:794-797.

21. Vlahov $D$, Junge $B$. The role of needle exchange programs in HIV prevention. Public Health Rep 1998; 113 (Suppl. 1):7580.

22. Crofts N, Nigro L, Oman K, Stevenson E, Sherman J. Methadone maintenance and hepatitis $C$ virus infection among injecting drug users. Addiction 1997; 92:999-1005.

23. Martinez-Sierra C, Arizcorreta A, Diaz F, Roldan $R$ Martin-Herrera L, Perez-Guzman E, Giron-Gonzalez JA. Progression of chronic hepatitis $\mathrm{C}$ to liver fibrosis and cirrhosis in patients coinfected with hepatitis $C$ virus and human immunodeficiency virus. Clin Infect Dis 2003 : 36:491-498.

24. Benhamou Y, Bochet M, Di Martino V, Charlotte F, Azria F, Coutellier $A$, et al. Liver fibrosis progression in human immunodeficiency virus and hepatitis $C$ virus coinfected patients. The Multivirc Group. Hepatology 1999; 30:10541058.

25. Thio CL, Seaberg EC, Skolasky R Jr, hair J, Visscher B, Munoz $A$, et al. HIV-1, hepatitis B virus, and risk of liver-related mortality in the Multicenter Cohort Study (MACS). Lancet 2002; 360:1921-1926.

26. Hestad K, Updike M, Selnes OA, Royal W III. Cognitive sequelae of repeated head injury in a population of intravenous drug users. Scand / Psychol 1995; 36:246-255.

27. Selnes OA Galai N, McArthur JC Cohn S, Royal W III, Esposito D, Vlahov D. HIV infection and cognition in intravenous drug users: long-term follow-up. Neurology 1997; 48:223230 .

28. Gamaleya N, Tagliaro F, Parshin A, Vrublevskii A, Bugari G, Dorizzi $R$, et al. Immune response to opiates: new findings in heroin addicts investigated by means of an original enzyme immunoassay and morphine determination in hair. Life $\mathrm{SCl}$ 1993; 53:99-105.

29. Vlahov D, Anthony JC, Munoz A, Margolick J, Nelson KE Celentano DD, et al. The ALIVE study, a longitudinal study of HIV-1 infection in intravenous drug users: description of methods and characteristics of participants. NIDA Res Monogr 1991; 109:75-100.

30. Limdi JK, Hyde GM. Evaluation of abnormal liver function tests. Postgrad Med J 2003; 79:307-312.

31. Corti MC, Guralnik JM, Salive ME, Sorkin JD. Serum albumin level and physical disability as predictors of mortality in older persons. JAMA 1994; 272:1036-1042.

32. Glesby MJ, Hoover DR. Survivor treatment selection bias in observational studies: examples from the AIDS literature. Ann Intern Med 1996; 124:999-1005.
33. Yeni PG, Hammer SM, Carpenter CC, Cooper DA, Fischl MA, Gatell JM et al. Antiretroviral treatment for adult HIV infection in 2002: updated recommendations of the International AIDS Society - USA Panel. JAMA 2002; 288:222-235.

34. Lamarca R, Alonso J, Gomez G, Munoz A. Left-truncated data with age as time scale: an alternative for survival analysis in the elderly population. I Gerontol A Biol Sci Med Sci 1998; 53:M337-M343.

35. Robins JM, Hernan MA, Brumback B. Marginal structural models and causal inference in epidemiology. Epidemiology 2000; 11:550-560.

36. Cole SR Hernan MA, Robins IM, Anastos K, Chmiel J Detels R, et al. Effect of highly active antiretroviral therapy on time to acquired immunodeficiency syndrome or death using marginal structural models. Am / Epidemiol 2003; 158:687-694.

37. Hernan MA, Brumback B, Robins JM. Marginal structural models to estimate the causal effect of zidovudine on the survival of HIV-positive men. Epidemiology 2000; 11:561570 .

38. Robins JM, Greenland S. Identifiability and exchangeability for direct and indirect effects. Epidemiology 1992 : 3:143-155.

39. Fisher LD, Lin DY. Time-dependent covariates in the Cox proportional-hazards regression model. Annu Rev Public Health 1999; 20:145-157.

40. McFarland W, Chen S, Hsu L, Schwarcz S, Katz M. Low socioeconomic status is associated with a higher rate of death in the era of highly active antiretroviral therapy, San Francisco. J Acquir Immune Defic Syndr 2003; 33:96-103.

41. Lin DY, Fischl MA, Schoenfeld DA. Evaluating the role of CD4-lymphocyte counts as surrogate endpoints in human immunodeficiency virus clinical trials. Stat Med 1993; 12:835-842.

42. Cole SR, Hernan MA. Fallibility in estimating direct effects. Int J Epidemiol 2002; 31:163-165.

43. Celentano DD, Munoz A, Cohn S, Nelson KE, Vlahov D. Drugrelated behavior change for HIV transmission among American injection drug users. Addiction 1994; 89:13091317.

44. Fox $\dot{R}$, Odaka NJ, Brookmeyer R, Polk BF. Effect of HIV antibody disclosure on subsequent sexual activity in homosexual men. AIDS 1987; 1:241-246.

45. McCusker J, Willis G, McDonald M, Sereti SM, Lewis BF, Sullivan JL. Community-wide HIV counselling and testing in central Massachusetts: who is retested and does their behavior change? / Commun Health 1996; 21:11-22.

46. Eichler MR, Ray SM, del Rio C. The effectiveness of HIV posttest counselling in determining healthcare-seeking behavior. AIDS 2002; 16:943-945.

47. McCusker J, Willis G, McDonald M, Lewis BF, Sereti SM, Feldman ZT. Admissions of injection drug users to drug abuse treatment following HIV counseling and testing. Public Health Rep 1994: 109:212-218.

48. Brugal MT, Barrio G, De LF, Regidor E, Royuela L, Suelves JM. Factors associated with non-fatal heroin overdose: assessing the effect of frequency and route of heroin administration. Addiction 2002; 97:319-327.

49. Morrison CL. Loss of tolerance and overdose mortality with detoxification: deaths have been associated with interventions. BMJ 2003; 327:393.

50. Thomas DL, Shih JW, Alter HJ, Vlahov D, Cohn S, Hoover DR, et al. Effect of human immunodeficiency virus on hepatitis $\mathbf{C}$ virus infection among injecting drug users. J Infect Dis 1996; 174:690-695.

51. Di $M, V$, Rufat $P$, Boyer $N$, Renard P, Degos F, MartinotPeignoux $\mathrm{M}$, et al. The influence of human immunodeficiency virus coinfection on chronic hepatitis $C$ in injection drug users: a long-term retrospective cohort study. Hepatology 2001; 34:1193-1199.

52. Tobin $\mathrm{KE}$, Latkin $\mathrm{CA}$. The relationship between depressive symptoms and nonfatal overdose among a sample of drug users in Baltimore, Maryland. J Urban Health 2003; 80:220229.

53. Galea S, Coffin PO. Drug overdose: new insights, innovative surveillance, and promising interventions. J Urban Health 2003; 80:186-188.

54. Darke $S$, Hall W. The distribution of naloxone to heroin users. Addiction 1997; 92:1195-1199. 